\title{
AVIAN INFLUENZA
}

Chaudhary S. ${ }^{1}$, Pahwa V.K. ${ }^{2}$

1. Editor, Journal of Universal College of Medical Sciences \& Teaching Hospital, Bhairahawa, Nepal

2. Chief Editor, Journal of Universal College of Medical Sciences \& Teaching Hospital, Bhairahawa, Nepal

Avian influenza refers to the disease caused by infection with avian (bird) influenza (flu) Type A viruses. It was first identified in Italy in the early 1900s and is now known to exist worldwide. These viruses occur naturally and have been isolated from more than 100 different species of wild aquatic birds which act as reservoir. It can infect domestic poultry and other bird and animal species. Influenza pandemics are associated with high morbidity, excess mortality, and social and economic disruption. Avian influenza A viruses are classified into the following two categories: low pathogenic avian influenza (LPAI) A viruses, and highly pathogenic avian influenza (HPAI) A viruses. Infection of poultry with LPAI viruses may cause no disease or mild illness and may not be detected. Infection of poultry with HPAI viruses can cause severe disease with high mortality. Both HPAI and LPAI viruses can spread rapidly through poultry flocks. The majority of the wild birds from which these viruses have been recovered represent gulls, terns and shorebirds or waterfowl such as ducks, geese and swans. Wild aquatic birds can be infected with avian influenza A viruses in their intestines and respiratory tract, but usually do not get sick. However, avian influenza A viruses both HPAI and LPAI are very contagious among birds. HPAI virus infection in poultry (such as with HPAI H5 or HPAI $\mathrm{H} 7$ viruses) can cause disease that affects multiple internal organs with mortality up to $90-100 \%$, often within 48 hours. Infected birds can shed avian influenza A viruses in their saliva, nasal secretions, and feces. Susceptible birds become infected when they have contact with the virus as it is shed by infected birds. ${ }^{1}$ They can also become infected by coming in contact with surfaces that are contaminated with virus from infected birds. When $\mathrm{H} 5$ or $\mathrm{H} 7$ avian influenza outbreaks occur in poultry, depopulation (or culling, also called "stamping out") of infected flocks is usually carried out. In addition surveillance of flocks that are nearby or linked to the infected flock(s), and quarantine of exposed flocks with culling if disease is detected, are the preferred control and eradication methods. Avian flu viruses do not normally infect humans. However, sporadic human infections with avian flu viruses have occurred. Avian influenza virus spreads in the air and in manure and there is no evidence that the virus can survive in well cooked meat. The most well known example is the avian influenza subtype H5N1 viruses currently circulating in poultry in parts of Asia and northeast Africa, which have caused human disease and deaths since 1997. Cumulative number of confirmed human cases for avian influenza A (H5N1) reported to WHO, 2003-2013 were 633 and there were 377 deaths. ${ }^{2}$ Other avian influenza subtypes, including H7N7 and H9N2, have also infected people. Some of these infections have been very severe and some have resulted in deaths, but many infections have been mild or even subclinical in humans.

January 16, 2009: H5N1 hits Nepal for first time. In a January 16 report to the World Organization for Animal Health (OIE), government officials in Nepal said the virus struck backyard poultry in a village in Jhapa district in the southeastern corner of Nepal. The first outbreak was confirmed in Kankarbhitta on January 16. 28,000 chickens were killed in the area to control the virus. ${ }^{3}$ Recent outbreak is noted in kathmandu and Bhaktapur district. The authorities in Nepal have declared parts of Kathmandu and Bhakatapur district as a "bird flu emergency zone" for three months to check an outbreak. Under the provision of emergency zone, all fowls including chickens will be culled, while feed, egg and excreta will be properly destroyed and disposed. Sales, supplies and farming of fowls will also be banned in the area. To control the spread of the virus, the government banned the import and transport of chicken in Kathmandu Valley for a week from August 1. Since then it has been conducting rapid tests on chickens imported into the Valley and those being transported out of poultry farms in the Valley. If these chickens are found infected with the influenza 'A' virus, the government teams immediately destroy them. Since the recent outbreak of bird flu, the government has killed over 1.56 million units of chicken and destroyed more than 1.20 million units of eggs in Kathmandu Valley, Chitwan, Kavre, Hetauda and other places around the country. ${ }^{4} \mathrm{CDC}$, United States Department of Agriculture (USDA), World Health Organization (WHO), World Organization for Animal Health (OIE), and Food and Agriculture Organization of the United Nations (FAO) conduct routine surveillance to monitor influenza viruses for changes that may have implications for animal and public health.

Mainly four influenza antiviral agents are available for treatment: amantadine, rimantadine, zanamivir and 
oseltamivir. Zanamivir and oseltamivir are active against both influenza $\mathrm{A}$ and $\mathrm{B}$ viruses. Amantadine and rimantadine are active against influenza $A$ viruses but not influenza $B$ viruses. In recent years, widespread adamantanes resistance among influenza A (H3N2) virus strains has made this class of medications less useful clinically. In addition, circulating 2009 H1N1 virus strains are resistant to adamantanes. Therefore, amantadine and rimantadine are not recommended for antiviral treatment or chemoprophylaxis of currently circulating influenza A virus strains. Zanamivir is approved for treatment of persons aged 7 years and older and approved for chemoprophylaxis of persons aged 5 years and older. Zanamivir is administered through oral inhalation by using a plastic device included in the medication package. Zanamivir has to be given $10 \mathrm{mg}$ ( 2 inhalations) twice daily for the treatment of influenza A and B, and $10 \mathrm{mg}$ (2 inhalations) once daily for chemoprophylaxis. Oseltamivir is approved for treatment of persons aged 2 weeks and older and for chemoprophylaxis of persons aged 1 year and older. Oseltamivir has to be given $75 \mathrm{mg}$ twice daily for the treatment of influenza A and B and for chemoprophylaxis $75 \mathrm{mg}$ once daily. Recommended duration for antiviral treatment is 5 days. Longer treatment courses can be considered for patients who remain severely ill after 5 days of treatment. Recommended duration of post-exposure chemoprophylaxis for high-risk patients is 7 days after the most recent known exposure if chemoprophylaxis can be started within 48 hours of exposure; however, early treatment if symptomatic is preferred. For control of outbreaks in long-term care facilities and hospitals, CDC recommends antiviral chemoprophylaxis for a minimum of 2 weeks and up to 1 week after the most recent known case was identified. ${ }^{5}$ Currently, the best way to prevent infection with avian influenza $A$ viruses is to avoid sources of exposure whenever possible. People who work with poultry or who respond to avian influenza outbreaks are advised to follow recommended biosecurity and infection control practices; these include use of appropriate personal protective equipment and careful attention to hand hygiene. In addition, HPAI poultry outbreak responders should adhere to guidance from $\mathrm{CDC}$ and $\mathrm{WHO}$ and receive seasonal influenza vaccination annually and take prophylactic antiviral medication during response. Seasonal influenza vaccination will not prevent infection with avian influenza A viruses, but can reduce the risk of co-infection with human and avian influenza A viruses. In particular, analysis of available HPAI $\mathrm{H} 5 \mathrm{~N} 1$ viruses circulating worldwide suggest that most viruses are susceptible to oseltamivir and zanamivir. However, some evidence of resistance to oseltamivir has been reported in HPAI H5N1 viruses isolated from some human HPAI H5N1 cases.

An outbreak of human infections with a new avian influenza A
(H7N9) virus was first reported in China by the World Health Organization on April 1, 2013. The virus was detected in poultry in China as well. During the outbreak, more than 130 human infections with H7N9 were reported. Many of the people infected with H7N9 reported contact with poultry. While some mild illness in human cases was seen, most patients had severe respiratory illness and 44 people died. Most concerning is the pandemic potential of this virus. CDC is following this situation closely and coordinating with domestic and international partners. ${ }^{6}$

\section{REFERENCES}

1. Avian Influenza in Birds. 2012 June 21 [cited 2013 Aug 21] Available from: URL: www.cdc.gov/flu/avianflu/avian-inbirds.htm

2. WHO. Influenza at the human-animal interface: Summary and assessment as of 4 July 2013. 2013 July 4 [cited 2013 Aug 21]; Available from: URL: www.who.int/influenza/ human_animal_interface/Influenza_Summary_IRA_HA_int erface_03July13.pdf

3. Bird Flu Strikes Nepal. Nepal News.Net. 2009 February 21[cited 2013 Aug 21]; Available from: URL: http://www.nepalnews.net/story/469479

4. Chicken Meat, Egg Demand Drops. thepoultrysite.com. 2013 August 23[cited 2013 Aug 25]; Available from: URL: www.thepoultrysite.com/poultrynews/29861/chicken-meat

5. Centers for Disease Control and Prevention. Antiviral Agents for the Treatment and Chemoprophylaxis of Influenza Recommendations of the Advisory Committee on Immunization Practices (ACIP). MMWR 2011 January 21;60(1);1-28.

6. Avian Influenza A (H7N9) Virus. 2013 Aug 13 [cited 2013 Aug 21]; Available from: URL: www.cdc.gov/flu/avianflu/h7n9virus.htm/ 